\title{
The Structure of Linear Extension Operators for $C^{m}$
}

\section{Charles Fefferman}

\begin{abstract}
For any subset $E \subset \mathbb{R}^{n}$, let $C^{m}(E)$ denote the Banach space of restrictions to $E$ of functions $F \in C^{m}\left(\mathbb{R}^{n}\right)$. It is known that there exist bounded linear maps $T: C^{m}(E) \longrightarrow C^{m}\left(\mathbb{R}^{n}\right)$ such that $T f=f$ on $E$ for any $f \in C^{m}(E)$. We show that $T$ can be taken to have a simple form, but cannot be taken to have an even simpler form.
\end{abstract}

\section{Statement of Results}

Fix $m, n \geq 1$, let $E \subset \mathbb{R}^{n}$ be given, and let $C^{m}(E)=\left\{\left.F\right|_{E}: F \in C^{m}\left(\mathbb{R}^{n}\right)\right\}$, with norm

$$
\|f\|_{C^{m}(E)}=\inf \left\{\|F\|_{C^{m}\left(\mathbb{R}^{n}\right)}: F \in C^{m}\left(\mathbb{R}^{n}\right) \text { and }\left.F\right|_{E}=f\right\} .
$$

Here, as usual, $C^{m}\left(\mathbb{R}^{n}\right)$ denotes the space of $m$ times continuously differentiable $F: \mathbb{R}^{n} \longrightarrow \mathbb{R}$, for which the norm

$$
\|F\|_{C^{m}\left(\mathbb{R}^{n}\right)}=\max _{|\alpha| \leq m} \sup _{x \in \mathbb{R}^{n}}\left|\partial^{\alpha} F(x)\right|
$$

is finite. A linear extension operator for $C^{m}(E)$ is a bounded linear map $T: C^{m}(E) \longrightarrow C^{m}\left(\mathbb{R}^{n}\right)$, such that $\left.T f\right|_{E}=f$ for all $f \in C^{m}(E)$.

Given $E \subset \mathbb{R}^{n}$, there exists a linear extension operator for $C^{m}(E)$. See $[17]$ for a proof, and $[1, \ldots, 29]$ for related work going back to Whitney. In particular, Merrien [20] constructed linear extension operators for $C^{m}(E)$ when $E \subset \mathbb{R}^{1}$, and Bromberg [3] constructed linear extension operators for $C^{1}(E)$ when $E \subset \mathbb{R}^{n}$. The existence of linear extension operators for $C^{m}(E)$ was explicitly conjectured by Brudnyi and Shvartsman in [9].

2000 Mathematics Subject Classification: 41A05, 41A45.

Keywords: Extension operators, Whitney's extension problem. 
The purpose of this paper is to examine what a linear extension operator for $C^{m}(E)$ might look like. For arbitrary finite $E$, we showed in [11] that $C^{m}(E)$ admits an extension operator of bounded "depth". We recall the relevant definition from [11], in a slightly weakened form.

Let $s \geq 1$ be an integer, and let $T: C^{m}(E) \longrightarrow C^{m}\left(\mathbb{R}^{n}\right)$ be a linear map. Then we say that $T$ has depth $s$ if, for every $x^{0} \in \mathbb{R}^{n}$, there exist $x_{1}, \ldots, x_{s} \in E$ and $\lambda_{1}, \ldots, \lambda_{s} \in \mathbb{R}$, such that

$$
T f\left(x^{0}\right)=\sum_{i=1}^{s} \lambda_{i} f\left(x_{i}\right) \text { for all } f \in C^{m}(E) .
$$

From [11], we have the following result.

Theorem 1. Given $m \geq 1$ and $E \subset \mathbb{R}^{n}$ finite, there exists an extension operator $T: C^{m}(E) \longrightarrow C^{m}\left(\mathbb{R}^{n}\right)$ with norm at most $C$ and depth at most s; here, $C$ and $s$ depend only on $m$ and $n$.

One might be tempted to believe that the hypothesis of finite $E$ can be dropped from Theorem 1. The following result dashes this hope.

Theorem 2. There exists a countable compact set $E \subset \mathbb{R}^{2}$, for which $C^{1}(E)$ admits no extension operator of finite depth.

We prove this result in Section 1 below, by exhibiting an explicit E. Our set $E$ is very close to a counterexample given by Glaeser in [18].

Despite Theorem 2, we can get a positive result by modifying the notion of "depth". We prepare the way with the following definitions.

A "one-point differential operator on $C^{m}\left(\mathbb{R}^{n}\right)$ " is a linear functional on $C^{m}\left(\mathbb{R}^{n}\right)$ of the form

(1) $\mathcal{D}: F \mapsto \sum_{|\alpha| \leq m} a_{\alpha} \partial^{\alpha} F\left(x^{0}\right)$, with $x^{0} \in \mathbb{R}^{n}$ and $a_{\alpha} \in \mathbb{R}(|\alpha| \leq m)$.

Next, let $E \subset \mathbb{R}^{n}$, and let $\mathcal{D}$ be as in (1). We say that $\mathcal{D}$ is a "one-point differential operator on $C^{m}(E)$ ", provided we have

(2) $\mathcal{D} F=0$ whenever $F \in C^{m}\left(\mathbb{R}^{n}\right)$ and $\left.F\right|_{E}=0$.

Evidently, if (1) and (2) hold, then we obtain a linear functional on $C^{m}(E)$, by mapping $f \in C^{m}(E)$ to $\mathcal{D} F$, for any $F \in C^{m}\left(\mathbb{R}^{n}\right)$ with $\left.F\right|_{E}=f$. Abusing notation, we denote this functional by $f \mapsto \mathcal{D} f$.

As a trivial example, suppose $E$ is an embedded sub-manifold in $\mathbb{R}^{n}$. Then any tangent vector $X \in T_{x^{0}} E$ is a one-point differential operator on $C^{1}(E)$. 
The paper [2] of Bierstone-Milman-Pawłucki shows how to find all possible one-point differential operators on $C^{m}(E)$ for an arbitrary, given $E \subset \mathbb{R}^{n}$. (See also [13].)

Now let $T: C^{m}(E) \longrightarrow C^{m}\left(\mathbb{R}^{n}\right)$ be a linear map and let $s \geq 1$. Then we say that $T$ has "breadth" $s$ if, given any one-point differential operator $\mathcal{D}$ on $C^{m}\left(\mathbb{R}^{n}\right)$, there exist one-point differential operators $\mathcal{D}_{1}, \ldots, \mathcal{D}_{s}$ on $C^{m}(E)$, such that

$$
\mathcal{D}(T f)=\sum_{i=1}^{s} \mathcal{D}_{i} f \text { for all } f \in C^{m}(E) .
$$

In particular, this implies that, for any $x^{0} \in \mathbb{R}^{n}$, we can express $T f\left(x^{0}\right)$ as a sum of at most $s$ terms of the form $\mathcal{D}_{i} f$, where $\mathcal{D}_{i}$ is a one-point differential operator on $C^{m}(E)$.

We are ready to state our positive result.

Theorem 3. Given $m \geq 1$ and $E \subset \mathbb{R}^{n}$, there exists an extension operator $T: C^{m}(E) \longrightarrow C^{m}\left(\mathbb{R}^{n}\right)$, with norm at most $C$ and breadth at most s; here, $C$ and $s$ depend only on $m$ and $n$.

The proof of Theorem 3 is accomplished by modifying the proof of the main result in [17], as explained in Section 2 below.

I am grateful to Gerree Pecht for LATEXing this paper with remarkable speed and accuracy.

\section{Proof of Theorem 2}

We exhibit the countable compact set $E \subset \mathbb{R}^{2}$ from Theorem 2. Let

(1) $P_{N, k}=\left(x_{N, k}, y_{N, k}\right)=\left(2^{-N}+10^{-N-k},(-1)^{k} \cdot 10^{-2 N-k}\right) \in \mathbb{R}^{2}$ for $N, k \geq 1$; and let

(2) $P_{N, \infty}=\left(2^{-N}, 0\right) \in \mathbb{R}^{2}$, for $N \geq 1$.

We define

(3) $E_{N}=\left\{P_{N, \infty}\right\} \cup\left\{P_{N, k}: k \geq 1\right\} \subset \mathbb{R}^{2}$ for $N \geq 1$,

and we set

(4) $E=\{(0,0)\} \cup \bigcup_{N \geq 1} E_{N}$.

Note that the $E_{N}$ are pairwise disjoint. As promised, $E$ is a countable compact subset of $\mathbb{R}^{2}$.

To show that $C^{1}(E)$ admits no extension operators of finite depth, we use the following three properties of $E$. 
Lemma 1. Let $\tilde{E} \subset E$, and suppose $\tilde{E} \cap E_{N}$ is finite for each $N \geq 1$. Then $\tilde{E} \subset\left\{(x, y) \in \mathbb{R}^{2}: y=\psi(x)\right\}$ for some function $\psi \in C^{1}(\mathbb{R})$.

Lemma 2. Let $s \geq 1$, and let $\left(P_{1}^{n}, \ldots, P_{s}^{n}\right)$ be a sequence of $s$-tuples of points of $E$. Then there exist an integer $N_{0} \geq 1$ and an increasing infinite sequence $\left(n_{\nu}\right)_{\nu \geq 1}$, such that $\left\{P_{i}^{n_{\nu}}: \nu \geq 1,1 \leq i \leq s\right\} \cap E_{N}$ is finite for each $N \geq N_{0}$.

Lemma 3. Let $F \in C^{1}\left(\mathbb{R}^{2}\right)$. If $F=0$ on $E$, then $\nabla F(0,0)=0$.

Assume these three lemmas for the moment, and suppose $T: C^{1}(E) \longrightarrow$ $C^{1}\left(\mathbb{R}^{2}\right)$ is an extension operator of depth $s$. We will derive a contradiction.

For $n \geq 1$, let

(5) $Q^{n}=\left(0, \frac{1}{n}\right) \in \mathbb{R}^{2}$.

Since $T$ has depth $s$, there exist points $P_{1}^{n}, \ldots, P_{s}^{n} \in E$ and coefficients $\lambda_{1}^{n}, \ldots, \lambda_{s}^{n} \in \mathbb{R}$, such that

$$
T f\left(Q^{n}\right)=\sum_{i=1}^{s} \lambda_{i}^{n} f\left(P_{i}^{n}\right) \text { for } f \in C^{1}(E), n \geq 1 .
$$

In particular, for each $n \geq 1$, we have

(6) $T f\left(Q^{n}\right)=0$ whenever $f \in C^{1}(E)$ with $f\left(P_{1}^{n}\right)=\cdots=f\left(P_{s}^{n}\right)=0$.

We apply Lemma 2 to the $s$-tuples $\left(P_{1}^{n}, \ldots, P_{s}^{n}\right), n \geq 1$.

Let $N_{0}$ and $\left(n_{\nu}\right)_{\nu \geq 1}$ be as in Lemma 2. We define sets

(7) $\hat{E}=\left\{P_{i}^{n_{\nu}}: \nu \geq 1,1 \leq i \leq s\right\}$,

(8) $E^{\#}=\left\{P \in \hat{E}: P \in E_{N}\right.$ for some $\left.N<N_{0}\right\}$, and

(9) $\tilde{E}=\hat{E} \backslash E^{\#}$.

The set $\tilde{E} \cap E_{N}$ is finite for $N \geq N_{0}$ (by Lemma 2), and empty for $N<N_{0}$ (by (8) and (9)). Hence, Lemma 1 applies, and there exists $\psi \in C^{1}(\mathbb{R})$ such that

(10) $y=\psi(x)$ for all $(x, y) \in \tilde{E}$.

Now let $\theta(x, y)$ be a smooth cutoff function on $\mathbb{R}^{2}$, equal to one in a neighborhood of the origin, and equal to zero on $E_{N}$ for $N<N_{0}$. We define

(11) $F(x, y)=\theta(x, y) \cdot[y-\psi(x)]$ for $(x, y) \in \mathbb{R}^{2}$, and

(12) $f=\left.F\right|_{E} \in C^{1}(E)$.

The functions $F$ and $T f$ both belong to $C^{1}\left(\mathbb{R}^{2}\right)$, and are both equal to $f$ on $E$. Hence, Lemma 3 gives

(13) $\nabla(T f)(0,0)=\nabla F(0,0)$. 
On the other hand, we can compute $\frac{\partial}{\partial y}(T f)(0,0)$ and $\frac{\partial}{\partial y} F(0,0)$, and they will turn out to be unequal.

In fact, we have $F=0$ on $\tilde{E}$ thanks to (10), (11); and $F=0$ on $E^{\# \text {, }}$ since $\theta=0$ on $E_{N}$ for $N<N_{0}$. (See (8), (11).) Thus, $F=0$ on $\hat{E}$, hence $f=0$ on $\hat{E}$, and therefore $\operatorname{Tf}\left(Q^{n_{\nu}}\right)=0$ for $\nu \geq 1$, thanks to (6) and (7).

Recalling (5), we conclude that

(14) $\frac{\partial}{\partial y}(T f)(0,0)=0$.

However, since $\theta=1$ in a neighborhood of the origin, the definition (11) yields (15) $\frac{\partial}{\partial y} F(0,0)=1$.

Thus, $\frac{\partial}{\partial y}(T f)(0,0)$ and $\frac{\partial}{\partial y} F(0,0)$ are distinct, as claimed.

This contradicts (13), showing that $C^{1}(E)$ cannot have an extension operator of depth $s$.

To complete the proof of Theorem 2, it remains to establish Lemmas 1, 2 and 3 . We begin with the following elementary result, which will be used in the proof of Lemma 1.

Proposition. Given $M \geq 1$, there exists $\psi_{M} \in C^{1}(\mathbb{R})$, with

(16) supp $\psi_{M} \subset(0,1)$,

(17) $\psi_{M}\left(10^{-k}\right)=(-1)^{k} \cdot 10^{-k}$ for $1 \leq k \leq M$, and

(18) $\left\|\psi_{M}\right\|_{C^{1}(\mathbb{R})} \leq C$, with $C$ independent of $M$.

Proof. Fix smooth functions $\theta, \tilde{\theta}$ on $\mathbb{R}$, with $\theta(x)=0$ for $x \leq 1 / 2, \theta(x)=1$ for $x \geq 1, \tilde{\theta}(x)=1$ for $|x| \leq 1 / 2, \tilde{\theta}(x)=0$ for $|x| \geq 2 / 3$. One checks easily that

$$
\psi_{M}(x)=\theta\left(10^{M} x\right) \cdot \tilde{\theta}(x) \cdot x \cos \left(\pi \log _{10}|x|\right)
$$

satisfies all the conditions asserted in the proposition.

Proof of Lemma 1. For each $N \geq 1$, pick

(19) $M_{N}>\max \left\{k: P_{N, k} \in \tilde{E}\right\}$.

We can do this, since $\tilde{E} \cap E_{N}$ is assumed finite. Define

(20) $\psi(x)=\sum_{N \geq 1} 10^{-2 N} \psi_{M_{N}}\left(10^{N} \cdot\left[x-2^{-N}\right]\right)$ for $x \in \mathbb{R}$,

with $\psi_{M_{N}}$ as in the Proposition.

Each summand in (20) is a $C^{1}$ function on $\mathbb{R}$, with the $N^{\text {th }}$ summand having $C^{1}$ norm at most $C \cdot 10^{-N}$. (This follows easily from (18).) Hence, $\psi \in C^{1}(\mathbb{R})$. 
From (1) and (17), we have

$$
10^{-2 N} \psi_{M_{N}}\left(10^{N} \cdot\left[x_{N, k}-2^{-N}\right]\right)=10^{-2 N} \psi_{M_{N}}\left(10^{-k}\right)=(-1)^{k} \cdot 10^{-2 N-k}
$$

for $1 \leq k \leq M_{N}$. Hence, (1) and (19) yield

(21) $10^{-2 N} \psi_{M_{N}}\left(10^{N} \cdot\left[x_{N, k}-2^{-N}\right]\right)=y_{N, k}$ whenever $P_{N, k} \in \tilde{E}$.

On the other hand, (16) implies easily that

(22) $10^{-2 N^{\prime}} \psi_{M_{N^{\prime}}}\left(10^{N^{\prime}} \cdot\left[x_{N, k}-2^{-N^{\prime}}\right]\right)=0$ whenever $N^{\prime} \neq N\left(N, N^{\prime}, k \geq 1\right)$.

Putting (21), (22) into (20), we see that

(23) $\psi\left(x_{N, k}\right)=y_{N, k}$ whenever $P_{N, k}=\left(x_{N, k}, y_{N, k}\right) \in \tilde{E}$.

For $(x, y)=P_{N, \infty}$ or $(0,0)$, we have $y=0$, and all the summands in (20) are equal to zero, thanks to (16). Hence,

(24) $\psi(x)=y$ whenever $(x, y)=P_{N, \infty}$ or $(0,0)$.

From (23), (24) and (3), (4), we conclude that $\psi(x)=y$ for all $(x, y) \in \tilde{E}$, since $\tilde{E} \subset E$. The proof of Lemma 1 is complete.

Proof of Lemma 2. For $n \geq 1$, let $\mathcal{P}^{n}$ be the set

(25) $\mathcal{P}^{n}=\left\{P_{1}^{n}, \ldots, P_{s}^{n}\right\}$.

Suppose $\mathcal{N}$ is any set of positive integers. We say that $\mathcal{N}$ is a "sink" if there are infinitely many $n \geq 1$ for which $\mathcal{P}^{n}$ intersects $E_{N}$ for each $N \in \mathcal{N}$. The empty set is a sink. On the other hand, no sink can have more than $s$ elements, since the $E_{N}$ are pairwise disjoint and the $\mathcal{P}^{n}$ have cardinality at most $s$. Hence there exists a sink $\overline{\mathcal{N}}$ of maximal cardinality. Thus,

(26) The set $A=\left\{n \geq 1: \mathcal{P}^{n}\right.$ intersects $E_{N}$ for each $\left.N \in \overline{\mathcal{N}}\right\}$ is infinite (since $\overline{\mathcal{N}}$ is a sink)

and

(27) Given $N \geq 1$ not belonging to $\overline{\mathcal{N}}$, there are at most finitely many $n \in A$ for which $\mathcal{P}^{n}$ intersects $E_{N}$. (Otherwise, $\overline{\mathcal{N}} \cup\{N\}$ would be a sink, contradicting the maximal cardinality of $\overline{\mathcal{N}}$.)

In view of $(26)$, we can write

(28) $A=\left\{n_{1}, n_{2}, n_{3}, \ldots\right\}$

for an infinite increasing sequence $\left(n_{\nu}\right)_{\nu \geq 1}$.

Since $\overline{\mathcal{N}}$ is a sink, it has at most $s$ elements. Hence we can pick an integer $N_{0} \geq 1$ such that

(29) $N_{0}>N$ for all $N \in \overline{\mathcal{N}}$. 
From (27), (28), (29), we learn the following:

(30) Given $N \geq N_{0}$, there are at most finitely many $\nu$ for which $\mathcal{P}^{n_{\nu}}$ intersects $E_{N}$.

From (25) and (30), we obtain the conclusion of Lemma 2.

Proof of Lemma 3. Let $F \in C^{1}\left(\mathbb{R}^{2}\right)$, with $F=0$ on $E$. Fix $N \geq 1$, and note that $F\left(P_{N, \infty}\right)=F\left(P_{N, k}\right)=0$ for $k \geq 1$. Consequently,

(31) $0=\lim _{\substack{k \rightarrow \infty \\(k \text { even })}}\left[\frac{F\left(P_{N, k}\right)-F\left(P_{N, \infty}\right)}{10^{-N-k}}\right]=\left(\frac{\partial F}{\partial x}+10^{-N} \frac{\partial F}{\partial y}\right)\left(P_{N, \infty}\right)$ and

(32) $0=\lim _{\substack{k \rightarrow \infty \\(k \text { odd })}}\left[\frac{F\left(P_{N, k}\right)-F\left(P_{N, \infty}\right)}{10^{-N-k}}\right]=\left(\frac{\partial F}{\partial x}-10^{-N} \frac{\partial F}{\partial y}\right)\left(P_{N, \infty}\right)$.

(See $(1) \ldots(4)$.)

From (31) and (32), we learn that $\nabla F\left(P_{N, \infty}\right)=0$. Taking the limit as $N \rightarrow \infty$, we conclude that $\nabla F(0,0)=0$, proving Lemma 3 .

We have now established Lemmas 1, 2 and 3. Since we reduced Theorem 2 to those lemmas, the proof of Theorem 2 is complete.

It is an amusing exercise to construct a linear extension operator for $C^{1}(E)$ with $E \subset \mathbb{R}^{2}$ given by (1)...(4).

\section{Sketch of Proof of Theorem 3}

We recall the main result of [17], then explain how to modify it to prove Theorem 3. We begin with some notation and definitions.

We write $\mathcal{R}_{x}$ for the ring of $m$-jets of smooth real-valued functions at $x \in \mathbb{R}^{n}$. For $F \in C^{m}\left(\mathbb{R}^{n}\right)$ and $x \in \mathbb{R}^{n}$, we write $J_{x}(F)$ to denote the $m$-jet of $F$ at $x$.

Let $E \subset \mathbb{R}^{n}$ be compact. For each $x \in E$, suppose we are given an $m$-jet $f(x) \in \mathcal{R}_{x}$ and an ideal $I(x)$ in $\mathcal{R}_{x}$. Then $(f(x)+I(x))_{x \in E}$ is called a "family of cosets". (We allow the possibilities $I(x)=\{0\}$ and $I(x)=\mathcal{R}_{x}$.) The family of cosets $(f(x)+I(x))_{x \in E}$ is called "Glaeser stable" if it satisfies the following condition: Given $x_{0} \in E$ and $P_{0} \in f\left(x_{0}\right)+I\left(x_{0}\right)$, there exists $F \in C^{m}\left(\mathbb{R}^{n}\right)$ such that $J_{x_{0}}(F)=P_{0}$, and $J_{x}(F) \in f(x)+I(x)$ for all $x \in E$.

More generally, suppose $\Xi$ is a vector space, and again let $E \subset \mathbb{R}^{n}$ be compact. For each $x \in E$, suppose we are given a linear map $\xi \mapsto f_{\xi}(x)$ from $\Xi$ into $\mathcal{R}_{x}$, and an ideal $I(x)$ in $\mathcal{R}_{x}$. Then we call $\left(f_{\xi}(x)+I(x)\right)_{x \in E, \xi \in \Xi}$ a "family of cosets depending linearly on $\xi \in \Xi$ ". We say that $\left(f_{\xi}(x)+\right.$ $I(x))_{x \in E, \xi \in \Xi}$ is "Glaeser stable" if, for each fixed $\xi \in \Xi$, the family of cosets $\left(f_{\xi}(x)+I(x)\right)_{x \in E}$ is Glaeser stable. 
These notions arise naturally in $[16,17]$, and we refer the reader to those papers for the motivation. The main result of [17] is as follows.

Theorem 4. Let $\Xi$ be a vector space, with seminorm $|\cdot| \cdot \operatorname{Let}\left(f_{\xi}(x)+\right.$ $I(x))_{x \in E, \xi \in \Xi}$ be a Glaeser stable family of cosets depending linearly on $\xi \in \Xi$.

Assume that for each $\xi \in \Xi$ with $|\xi| \leq 1$, there exists $F \in C^{m}\left(\mathbb{R}^{n}\right)$, with $\|F\|_{C^{m}\left(\mathbb{R}^{n}\right)} \leq 1$, and $J_{x}(F) \in f_{\xi}(x)+I(x)$ for all $x \in E$.

Then there exists a linear map $\xi \mapsto F_{\xi}$, from $\Xi$ into $C^{m}\left(\mathbb{R}^{n}\right)$, such that

(A) $J_{x}\left(F_{\xi}\right) \in f_{\xi}(x)+I(x)$ for all $x \in E, \xi \in \Xi$; and

(B) $\left\|F_{\xi}\right\|_{C^{m}\left(\mathbb{R}^{n}\right)} \leq C|\xi|$ for all $\xi \in \Xi$, with $C$ depending only on $m$ and $n$.

This result easily implies the existence of extension operators for $C^{m}(E)$. To prove Theorem 3, we modify Theorem 4 by introducing the notion of " $s$ admissible" operators, which we now explain.

Let $\hat{\Xi}$ be a set of (real) linear functionals on the linear space $\Xi$, and let $s \geq 1$ be an integer. Then:

- A linear functional on $\Xi$ will be called "s-admissible" (with respect to $\hat{\Xi}$ ) if it can be written as a linear combination of at most $s$ elements of $\hat{\Xi}$.

- A linear map $T$ from $\Xi$ to a finite-dimensional vector space $V$ is called "s-admissible" (with respect to $\hat{\Xi}$ ) if, for every linear functional $\lambda$ on $V$, the linear functional $\lambda \circ T$ on $\Xi$ is $s$-admissible.

- A linear map $T: \Xi \longrightarrow C^{m}\left(\mathbb{R}^{n}\right)$ will be called " $s$-admissible" (with respect to $\hat{\Xi}$ ) if, for every $x \in \mathbb{R}^{n}$, the map $\xi \mapsto J_{x}(T \xi)$ is $s$-admissible as a map from $\Xi$ to $\mathcal{R}_{x}$.

Our modification of Theorem 4 is as follows.

Theorem 5. Let $\Xi$ be a vector space, with seminorm $|\cdot|$, let $\hat{\Xi}$ be a set of linear functionals on $\Xi$, and let $s \geq 1$ be an integer. Let $\left(f_{\xi}(x)+I(x)\right)_{x \in E, \xi \in \Xi}$ be a Glaeser stable family of cosets depending linearly on $\xi \in \Xi$.

Assume that the map $\xi \mapsto f_{\xi}(x)$, from $\Xi$ into $\mathcal{R}_{x}$, is s-admissible with respect to $\hat{\Xi}$, for each $x \in E$.

Assume also that, for each $\xi \in \Xi$ with $|\xi| \leq 1$, there exists $F \in C^{m}\left(\mathbb{R}^{n}\right)$, with

$$
\|F\|_{C^{m}\left(\mathbb{R}^{n}\right)} \leq 1 \text {, and } J_{x}(F) \in f_{\xi}(x)+I(x) \text { for all } x \in E .
$$

Then there exists a linear map $\xi \mapsto F_{\xi}$, from $\Xi$ into $C^{m}\left(\mathbb{R}^{n}\right)$, such that

(A) $J_{x}\left(F_{\xi}\right) \in f_{\xi}(x)+I(x)$ for all $x \in E, x \in \Xi$;

(B) $\left\|F_{\xi}\right\|_{C^{m}\left(\mathbb{R}^{n}\right)} \leq C|\xi|$ for all $\xi \in \Xi$, with $C$ depending only on $m$ and $n$;

(C) The map $\xi \mapsto F_{\xi}$ is $s^{\prime}$-admissible, with $s^{\prime}$ depending only on $s, m$ and $n$. 
We indicate briefly why Theorem 5 implies Theorem 3 and then we explain how the proof of Thm. 4 in [17] may be modified to prove Theorem 5 .

Reduction of Theorem 3 to Theorem 5 . To prove Theorem 3, we may assume that the set $E$ is compact. (In fact, for a general $E$, we may pass without difficulty to the closure of $E$, and then reduce matters to the case of closed, bounded $E$ by a partition of unity.)

For $E \subset \mathbb{R}^{n}$ compact, we make the following definitions.

- $\Xi=C^{m}(E)$.

- $|\xi|=2\|\xi\|_{C^{m}(E)}$ for $\xi \in \Xi$.

- $\widehat{\Xi}$ is the set of all one-point differential operators on $C^{m}(E)$.

For each $x \in E$ :

- $I(x)=\left\{J_{x}(F): F \in C^{m}\left(\mathbb{R}^{n}\right)\right.$ and $F=0$ on $\left.E\right\}$.

- $V(x)=$ any complementary subspace to $I(x)$ in $\mathcal{R}_{x}$.

- $\pi_{x}: \mathcal{R}_{x} \longrightarrow V(x)$ is the natural projection arising from the direct sum $\mathcal{R}_{x}=V(x) \oplus I(x)$.

Suppose $x \in E$ and $\xi \in \Xi$. Since $\xi \in \Xi$, there exists $F \in C^{m}\left(\mathbb{R}^{n}\right)$ with $\left.F\right|_{E}=\xi$. We define $f_{\xi}(x)=\pi_{x}\left(J_{x}(F)\right)$. This is independent of the choice of $F$. (In fact, suppose $F_{1}, F_{2} \in C^{m}\left(\mathbb{R}^{n}\right)$, with $\left.F_{i}\right|_{E}=\xi$. Then $F_{1}-F_{2} \in C^{m}\left(\mathbb{R}^{n}\right)$ and $\left.\left(F_{1}-F_{2}\right)\right|_{E}=0$. Hence, $J_{x}\left(F_{1}-F_{2}\right) \in I(x)$, and therefore $\pi_{x}\left(J_{x}\left(F_{1}\right)-J_{x}\left(F_{2}\right)\right)=0$.)

One checks easily that the above $\Xi,|\cdot|, \widehat{\Xi}, I(x), f_{\xi}(x)$ satisfy the hypotheses of Theorem 5, with $s=1$. Hence, applying Theorem 5, we obtain a linear map $\xi \mapsto F_{\xi}$ from $\Xi$ into $C^{m}\left(\mathbb{R}^{n}\right)$, satisfying $(\mathrm{A}),(\mathrm{B}),(\mathrm{C})$.

From (A), we see that $\xi \mapsto F_{\xi}$ is an extension operator for $C^{m}(E)$. Conclusion (B) controls the norm of this extension operator, and conclusion $(\mathrm{C})$ tells us that it has breadth $s^{\prime}$, with $s^{\prime}$ depending only on $m$ and $n$. Thus, Theorem 3 is reduced to Theorem 5 .

Sketch of Proof of Theorem 5. We assume that the reader is familiar with our previous papers $[11, \ldots, 17]$. It is a long, routine exercise to follow the proof of Theorem 4, as given in [17], and note that at each step, we preserve $s^{\prime}$-admissibility (although $s^{\prime}$ may increase). ("Admissibility" will always be defined with respect to $\widehat{\Xi}$, given in the hypotheses of Theorem 5.) The highlights of this tedious exercise are as follows.

- For $E \subset \mathbb{R}^{n}$ compact, let $C_{\text {jet }}^{m}(E)$ be the space of families of jets $\vec{f}=$ $\left(f_{x}\right)_{x \in E}$, with $f_{x} \in \mathcal{R}_{x}$ for each $x \in E$, such that there exists $F \in$ $C^{m}\left(\mathbb{R}^{n}\right)$ satisfying

(1) $J_{x}(F)=f_{x}$ for each $x \in E$. 
The norm $\|\vec{f}\|_{C_{\mathrm{jet}}^{m}(E)}$ is defined as the infimum of $\|F\|_{C^{m}\left(\mathbb{R}^{n}\right)}$ over all $F \in C^{m}\left(\mathbb{R}^{n}\right)$ satisfying (1).

The proof of the standard Whitney extension theorem [19, 24, 25] gives an operator $T: C_{\text {jet }}^{m}(E) \longrightarrow C^{m}\left(\mathbb{R}^{n}\right)$, with the following properties.

(a) $\|T\| \leq C$, with $C$ depending only on $m$ and $n$.

(b) For $\vec{f}=\left(f_{x}\right)_{x \in E} \in C_{\text {jet }}^{m}(E)$, we have $J_{x}(T \vec{f})=f_{x}$ for each $x \in E$.

(c) For each $x_{0} \in \mathbb{R}^{n}$ there exist $x_{1}, \ldots, x_{k} \in E$ such that, as $\vec{f}=\left(f_{x}\right)_{x \in E}$ varies over $C_{\text {jet }}^{m}(E)$, the jet $J_{x_{0}}(T \vec{f})$ depends only on $f_{x_{1}}, \ldots, f_{x_{k}}$. Here, $k$ depends only on $m$ and $n$.

In view of (c), we have the following result.

Let $\xi \mapsto \vec{f}_{\xi}=\left(f_{x, \xi}\right)_{x \in E}$ be a linear map from $\Xi$ into $C_{\text {jet }}^{m}(E)$. Assume that $\xi \mapsto f_{x, \xi}$ is $s^{\prime}$-admissible, for each $x \in E$.

Then the map $\xi \mapsto T \vec{f}_{\xi}$ is $s^{\prime \prime}$-admissible from $\Xi$ into $C^{m}\left(\mathbb{R}^{n}\right)$, where $T$ is as above, and $s^{\prime \prime}$ depends only on $s^{\prime}, m, n$.

- Suppose we add to the hypotheses of Lemma 3.3 in [16] the assumption that $\xi \mapsto f_{\xi}(x)$ is $s^{\prime}$-admissible for each $x \in E$. (Here, $s^{\prime} \geq 1$ is given.) Then the map $\xi \mapsto \tilde{f}_{\xi}\left(x_{0}\right)$ in the conclusion of that lemma may be taken to be $s^{\prime \prime}$-admissible, with $s^{\prime \prime}$ depending only on $s^{\prime}, m, n, k^{\#}$. (That's because the $\tilde{f}_{\xi}\left(x_{0}\right)$ constructed in the proof of Lemma 3.3 in [16] depends on $\xi$ only through the $f_{\xi}(x)$ for $x \in \bar{S}$, where $\bar{S} \subset E$ has cardinality less than $k^{\#}$.) When we apply the above lemma in [17], we take $k^{\#}$ to depend only on $m$ and $n$.

Therefore, the property of $s^{\prime}$-admissibility (for some $s^{\prime}$ depending only on $m, n, s)$ is preserved when we apply Lemma 3.3 from [16].

- Whenever we applied Theorem 5 from [16] in the proof of Theorem 4, we now apply instead Theorem 8 from [16]. Note that the notion of "depth" in [16] differs from our present notion.

- For suitable $x \in E$, let $\operatorname{proj}_{x}: \mathcal{R}_{x} \longrightarrow \mathcal{R}_{x}$ be the linear map defined in Section 10 of [17]. If $\xi \mapsto g_{\xi}(x)$ is an $s^{\prime}$-admissible linear map from $\Xi$ into $\mathcal{R}_{x}$, then also $\xi \mapsto \operatorname{proj}_{x}\left(g_{\xi}(x)\right)$ is $s^{\prime}$-admissible. (This follows trivially from the definition of $s^{\prime}$-admissibility.)

- Suppose $F_{\xi}=\sum_{\nu} \theta_{\nu} \cdot F_{\xi}^{\nu}$ for $\xi \in \Xi$; and suppose that, for each $x \in \mathbb{R}^{n}$, we are given a finite set $\Omega(x)$, such that

$$
J_{x}\left(F_{\xi}\right)=\sum_{\nu \in \Omega(x)} J_{x}\left(\theta_{\nu} \cdot F_{\xi}^{\nu}\right) \text { for all } \xi \in \Xi .
$$

If $\xi \mapsto F_{\xi}^{\nu}$ is $s^{\prime}$-admissible for each $\nu$, and if $\Omega(x)$ has cardinality at most $k$ for each $x \in \mathbb{R}^{n}$, then $\xi \mapsto F_{\xi}$ is $s^{\prime \prime}$-admissible, with $s^{\prime \prime}=k \cdot s^{\prime}$. 
Finally, we can prove Theorem 5 by following the proof of Theorem 4 in [17], and using the above observations to keep track of $s^{\prime}$-admissibility of every operator and functional that enters the argument. We dispense with further details.

\section{References}

[1] Bierstone, E., Milman, P. and PawŁucki, W.: Differentiable functions defined on closed sets. A problem of Whitney. Invent. Math. 151 (2003), no. 2, 329-352.

[2] Bierstone, E., Milman, P. and PawŁucki, W.: Higher-order tangents and Fefferman's paper on Whitney's extension problem. Ann. of Math (2) 164 (2006), 361-370.

[3] Bromberg, S.: An extension in class $C^{1}$. Bol. Soc. Mat. Mex. (2) 27 (1982), no. 2, 35-44.

[4] Brudnyi, Y. and Shvartsman, P.: The traces of differentiable functions to subsets of $\mathbb{R}^{n}$ in Linear and complex Analysis. Lect. Notes in Math., 279-281. Spring-Verlag, 1994.

[5] Brudnyi, Y.: On an extension theorem. Funk. Anal. i Prilzhen. 4 (1970), 97-98; English transl. in Func. Anal. Appl. 4 (1970), 252-253.

[6] Brudyni, A. And Brudyni, Y.: Metric spaces with linear extensions preserving Lipschitz condition. Amer. Math. J. 129 (2007), no. 1, 217-314.

[7] Brudnyi, Y. And Shvartsman, P.: A linear extension operator for a space of smooth functions defined on closed subsets of $\mathbb{R}^{n}$. Dokl. Akad. Nauk SSSR 280 (1985), no. 2, 268-270.

[8] Brudnyi, Y. and Shvartsman, P.: Generalizations of Whitney's extension theorem. Internat. Math. Res. Notices 3 (1994), 129-139.

[9] Brudnyi, Y. and Shvartsman, P.: The Whitney problem of existence of a linear extension operator. J. Geom. Anal. 7 (1997), no. 4, 515-574.

[10] Brudnyi, Y. and Shvartsman, P.: Whitney's extension problem for multivariate $C^{1, w}$ functions. Trans. Amer. Math. Soc. 353 (2001), no. 6, $2487-2512$.

[11] Fefferman, C.: Interpolation and extrapolation of smooth functions by linear operators. Rev. Mat. Iberoamericana 21 (2005), no. 1, 313-348.

[12] C. Fefferman: A sharp form of Whitney's extension theorem. Ann. of Math. (2) 161 (2005), no. 1, 509-577.

[13] Fefferman, C.: Whitney's extension problem for $C^{m}$. Ann. of Math. (2) 164 (2006), no. 1, 313-359.

[14] Fefferman, C.: Whitney's extension problem in certain function spaces. Preprint.

[15] Fefferman, C.: A generalized sharp Whitney theorem for jets. Rev. Mat. Iberoamericana 21 (2005), no. 2, 577-688. 
[16] Fefferman, C.: Extension of $C^{m, \omega}$ smooth functions by linear operators. To appear in Rev. Mat. Iberoamericana.

[17] Fefferman, C.: $C^{m}$ extension by linear operators. To appear in Ann. of Math. (2) 166 (2007), no. 3.

[18] Glaeser, G.: Etudes de quelques algebres tayloriennes. J. Analyse Math. 6 (1958), 1-124.

[19] Malgrange, B.: Ideals of Differentiable Functions. Oxford Univ. Press, 1966.

[20] Merrien, J.: Prolongateurs de foncions differentiables d'une variable relle. J. Math. Pures Appl. (9) 45 (1966), 291-309.

[21] Shvartsman, P.: Lipshitz selections of multivalued mappings and traces of the Zygmund class of functions to an arbitrary compact. Dokl. Acad. Nauk SSSR 276 (1984), 559-562; English transl. in Soviet Math. Dokl. 29 (1984), 565-568.

[22] Shvartsman, P.: On traces of functions of Zygmund classes. Sibirskyi Mathem. J. 28 N5 (1987), 203-215; English transl. in Siberian Math. J. 28 (1987), 853-863.

[23] Shvartsman, P.: Lipschitz selections of set-valued functions and Helly's theorem. J. Geom. Anal. 12 (2002), no. 2, 289-324.

[24] Stein, E. M.: Singular Integrals and Differentiability Properties of Functions. Princeton Univ. Press, 1970.

[25] Whitney, H.: Analytic extensions of differentiable functions defined in closed sets. Trans. Amer. Math. Soc. 36 (1934), 63-89.

[26] Whitney, H.: Differentiable functions defined in closed sets I. Trans. Amer. Math. Soc. 36 (1934), 369-3897.

[27] Whitney, H.: Functions differentiable on the boundaries of regions. Ann. of Math. 35 (1934), 482-485.

[28] Zobin, N.: Whitney's problem on extendability of functions and an intrinsic metric. Adv. Math. 133 (1998), no. 1, 96-132.

[29] Zobin, N.: Extension of smooth functions from finitely connected planar domains. J. Geom. Anal. 93 (1999), 489-509.

Recibido: 14 de julio de 2005

Charles Fefferman

Department of Mathematics

Princeton University

Fine Hall, Washington Road

Princeton, New Jersey 08544

cf@math.princeton.edu 